\title{
Price-based Energy Control for V2G Networks in the Industrial Smart Grid
}

\author{
Rong Yu*, Jiefei Ding*, Weifeng Zhong*, Yan Zhang ${ }^{\dagger}$, Stein Gjessing ${ }^{\ddagger}$, Alexey Vinel ${ }^{\S}$, Magnus Jonsson ${ }^{\S}$ \\ * Guangdong University of Technology, China \\ $\dagger$ Simula Research Laboratory, Norway \\ $\ddagger$ University of Oslo, Norway \\ $\S$ Halmstad University, Sweden \\ Email: \{yurong,wf.zhong, jf.ding\}@gdut.edu.cn, yanzhang@simula.no \\ steing@ifi.uio.no, $\{$ alexey.vinel, magnus.jonsson $\} @$ hh.se
}

\begin{abstract}
The energy crisis and global warming call for a new industrial revolution in production and distribution of renewable energy. Distributed power generation will be well developed in the new smart electricity distribution grid, in which robust power distribution will be the key technology. In this paper, we present a new vehicle-to-grid (V2G) network for energy transfer, in which distributed renewable energy helps the power grid balance demand and supply. Plug-in hybrid electric vehicles (PHEVs) will act as transporters of electricity for distributed renewable energy dispatching. We formulate and analyze the V2G network within the theoretical framework of complex network. We also employ the generalized synchronization method to study the dynamic behavior of V2G networks. Furthermore, we develop a new pricebased energy control method to stimulate the PHEV's behavior of charging and discharging. Simulation results indicate that the V2G network can achieve synchronization and each region is able to balance energy supply and demand through price-based control.
\end{abstract}

Index Terms-Vehicle-to-grid networks, industrial smart grid, demand response management, energy control, renewable energy, complex networks, synchronization, plug-in hybrid electric vehicles, dynamic pricing.

\section{INTRODUCTION}

A smarter power grid is urgently needed to deal with the predominating situation when there is a shortage of electricity. In the new smart grid, distributed generation of electricity and plug-in hybrid electric vehicles (PHEVs) will be well developed. Renewable electricity generation technology is not sufficiently mature yet since power resources are unevenly distributed, power generation is subjected to disturbance, and the capacity is unstable.

Exploiting renewable energy in the smart grid poses several challenges. The first is to develop a hybrid power system which includes wind and solar thermal energy [1]. The second is to design a distributed system in the smart grid for renewable energy dispatching [2], [3]. Besides, vehicles that can connect to the grid, called gridable vehicles (GVs), have been proposed as the medium to transport power between the renewable energy plants and the power grid in order to reduce cost and emissions [4]. Moreover, the technology of vehicle-togrid (V2G) [5] has enhanced the energy interaction between the PHEVs and power grid, which leads to a situation where
PHEVs can be both consumers and suppliers in the smart grid, and eventually constitute the back-up power supply [6], [7].

It is envisioned that PHEVs will play a more important role in the smart grid than energy storage. The understanding of PHEVs mobility has been a fundamental question and challenging issue. On the other side, Complex network theory has been a powerful tool for describing and analyzing complex environments. The complex network theory has been widely used in many fields, such as the World-Wide-Web, the Internet, wireless networks, power networks and literature index systems [8]-[11], to structure and analyze various complex problems. The synchronization analysis method is efficient when studying the dynamic behavior of complex networks. There are three main kinds of synchronization: identical synchronization, phase synchronization and generalized synchronization [12]. The most popular class is identical synchronization which has been used to improve the utilization of frequency resources in wireless mesh networks [13].

In this paper, we develop a paradigm of V2G network in the smart grid for two purposes. The first is to view the PHEVs as the carriers of renewable energy in the smart grid. The second is to conduct the price-based synchronization control in order to achieve energy demand-supply balance among different regions. We have three major contributions in this paper.

1) We exploit the complex network theory to model and analyze the features and dynamic behaviors of the $\mathrm{V} 2 \mathrm{G}$ network. The model is flexible and may be extended for many practical scenarios.

2) Generalized synchronization is leveraged as a control method to manage energy dispatching, by which the V2G network will reach a balance state.

3) Energy transportation by PHEVs is a viable solution with low loss and flexible control. The solution enables the smart grid to maximize the use of geographical resources and promote the development of distributed energy generation.

The rest of this paper is organized as follows. Section II introduces the new paradigm of the V2G network with mobile 


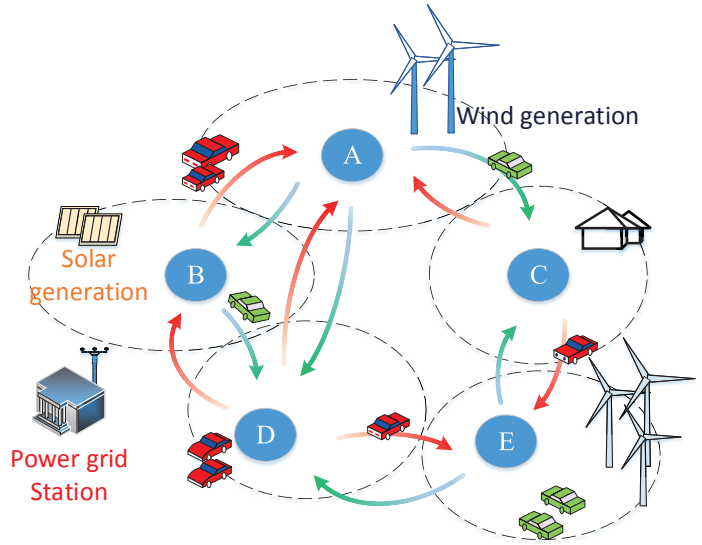

Fig. 1: The V2G network.

PHEVs. Section III presents the system model, in which the energy topology network and the activation function are given. In Section IV, the method of generalized synchronization is discussed. After that, the simulations are conducted in Section V. The conclusion is finally drawn in Section VI.

\section{THE V2G NETWORK}

As the demand for electricity is significantly increasing every year, traditional power supply is vulnerable as it is heavily dependent on the centralized control of the power grid. However, since medium-to-small size renewable energy generation plants have appeared throughout the world recently, distributed renewable energy has been considered as the promising solution for balancing energy supply and demand.

The V2G network is formed by the cooperation among distributed generation facilities, local batteries and PHEVs, which interacts with the power grid. Fig.1 shows the V2G architecture and its main components. The V2G network is modeled as a graph. Each geographic region in the V2G network is represented as a node in the graph. The energy distribution among regions is usually uneven. Some of the regions are equipped with renewable generation equipments. For instance, region $A$ has a wind generation plant and region $B$ has been installed with a photovoltaic unit. The layout of the renewable plants depends on the benefits of both the local resources and the electricity demand. In addition, the whole area is powered by the main power grid. The local batteries of each region are viewed as containers for energy storage. The source of the energy can be from the renewable energy power plants or/and from PHEVs discharging, and should be used according to the local energy demand. The mobility of the PHEVs is leveraged as the bridge connecting the power equipments in different regions into a complete energy system.

\section{A. Energy Distribution by PHEVs}

Smart grid is a complex dynamic system. For instance, the amount of available renewable energy, energy requirements and the number of PHEVs and their battery status are all

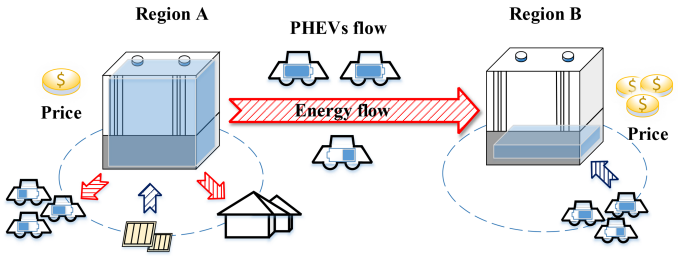

Fig. 2: The energy flow between region $A$ and region $B$.

changing from time to time in different locations. The V2G network should have a flexible structure that adapts to these changes. Without an appropriate dispatching strategy, unexpected situations may happen. In some regions, renewable energy may be abundant but the local batteries are over charged. Newly generated renewable energy will then be wasted if there is no storage left. In some other regions, local batteries have stored energy less than the demand. A peak period of energy demand will then create a high peak of power distribution, leading to the instability of grid.

The theory of complex network synchronization is intended to drive all nodes in the network to realize their synchronous work. The region that maintains a high electricity storage tends to move energy to a region in shortage, and hence achieves storage balance with the other regions. As depicted in Fig. 2, region $A$ sets a lower price than region $B$. This will cause PHEVs in region $A$ to charge more energy than region $B$. However, region $B$ adopts the opposite strategy so that PHEVs will discharge energy. Then, the PHEVs moving from region $A$ to region $B$ will bring an amount of electricity to region $B$, and at the same time the PHEVs that flow in the opposite direction will consume electricity in region $A$. The eventual consequence is that energy in region $A$ will be equivalently transported to region $B$ with the PHEVs' mobility. In the next section, we will build up an analytical model for the V2G network with PHEVs' mobility and energy control.

\section{System MOdEL}

\section{A. Complex Network Structure}

The V2G network is viewed as a graph that consists of $N \mathrm{~d}$ ifferent linearly coupled nodes. Each node represents the local batteries in one region. There are two main factors that connect to the energy states of local batteries: the internal state and the external state. The internal state indicates the fluctuation of the supply of renewable energy. The external state indicates the travel of the PHEVs and the battery consumption of one trip. Thus, the energy topology network is based on the general expression of complex network which is given by

$\dot{x_{i}}=f\left(x_{i}(t), n_{i}, \beta_{i}\right)+C \sum_{j=1}^{N} a_{j i} \Gamma x_{j}(t)-L \sum_{j=1}^{N} b_{j i} \Gamma x_{j}(t)$,

where $x_{i}(t)=\left(x_{i 1}(t), x_{i 2}(t), \cdots, x_{i m}(t)\right)^{T} \in R^{m}$ is the $m$ dimension state vector of node $i$ at time $t(i=1,2, \cdots, N)$. The state vector is desfined to represent the local energy state. $f(): R^{m} \rightarrow R^{m}$ is a continuously differentiable activation 
function with two parameters $n_{i}$ and $\beta_{i}$. Here, $n_{i}$ is the total number of PHEVs that are plugged in charging stations in region $i$ and $\beta_{i}$ is a constant related to original state. $C$ and $L$ indicate the coupling strength. The first parameter is related to the flow of PHEVs, thus, $C=1$. The second parameter is the mean value of energy consumption loss in one trip, $L=$ loss. The last two items in (1) are related to the external state. The configuration matrix $A=\left(a_{i j}\right)_{N \times N} \in R^{N \times N}$ is the statistics of traffic flows between nodes, which follows the rule: if there is a direct connection from node $i$ to node $j(i \neq j)$, the parameter of $a_{i j}$ is $k$, i.e., $a_{i j}=k$ when there are $k$ PHEVs departing from $i$ to $j$ during one time span after $t$. Otherwise, if there are no PHEV passed by, the parameter is zero, i.e., $a_{i j}=0$. The diagonal elements of matrix $A$ are defined as $a_{i i}=-\sum_{j=1, j \neq i}^{N} a_{i j}$, which is the total number of leaving PHEVs. For matrix $B, b_{i j}=a_{i j}, i \neq j$, while $b_{i i}$ denotes the number of trips where both the start and the end places are region $i$. Both matrix $A$ and matrix $B$ are coupling matrixes in (1). They are the asymmetrical matrixes. In this paper, we consider three-dimensional vector state (i.e., $m=3$ ) with $\Gamma$ the interval coupling matrix defined by $\Gamma=\left(\begin{array}{lll}0 & 0 & 0 \\ 0 & 1 & 0 \\ 0 & 0 & 0\end{array}\right)$.

\section{B. Activation Function}

The activation function is constructed by the principle of energy conservation. There are three interactional factors that represent the energy state of the local batteries. The first is the energy level of the local batteries, $x_{i 1}$, which reflects the energy demand and supply balance in the region $i$. Generally, the demand and supply balance is the state when electric facilities are running on the best efficiency state. In this paper, we employ a ratio $\eta$, and consider the situation in which the system will achieve its demand-supply balance on $\eta$ of the maximum load capability. The initial state of $x_{i 1}, x_{i 1}(0)$, can be written as

$$
\begin{aligned}
& x_{i 1}(0)=\eta \text { Load_max }_{i} \Delta t-\text { Load_t }_{i}, \\
& {\operatorname{Load} \_t_{i}}_{i}=\int_{\alpha}^{\beta} \operatorname{Load}_{i}(t) d t-\text { Battery }_{i}, \\
& \Delta t=\beta-\alpha,
\end{aligned}
$$

where Load_re ${ }_{i}$ is the total load in region $i$, which includes the real-time load $\operatorname{Load}_{\mathrm{i}}(\mathrm{t})$ and battery storage. The time slot is $\Delta t$ from $\alpha$ to $\beta$. The maximum load capability is represented

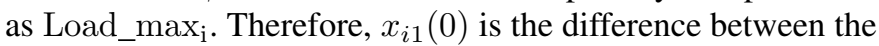
total load and the ideal load.

The second factor, $x_{i 2}$, is the total energy of PHEVs in region $i$. The third factor $x_{i 3}$ is the target state of charge (SOC) in region $i$. Region $i$ calculates the target SOC based on the load demand. The target SOC is reached by setting an appropriate price to control PHEVs behavior of charging or discharging. In other words, by setting a target SOC, we can balance the electricity demand in region $i$. For example, when region $i$ has redundant energy, a higher SOC can reduce the local price to attract PHEVs to charge more energy. Thus, $x_{i 3}$ will be increased by $k$ times of $x_{i 1}$. The ratio $k$ is a positive value. We describe $x_{i 3}$ by

$$
x_{i 3}=x_{i 3}+k x_{i 1} .
$$

The initial value of $x_{i 3}$, i.e., $x_{i 3}(0)$, is the average charging level of the PHEVs' batteries in region $i$. In the model, all PHEVs in region $i$ are considered in the state $x_{i 3}$. We have

$$
x_{i 2}=n_{i} x_{i 3},
$$

where $n_{i}$ is the total number of PHEVs that are plugged in charging stations in region $i$.

The energy from $x_{i 1}$ can be consumed or recharged in two ways: from visiting PHEVs or from local PHEVs. Since visiting energy flow only effects $x_{i 2}$, the difference between $x_{i 2}$ and $n_{i} x_{i 3}$ is the external influence by visiting PHEVs. Besides, the energy fluctuation caused by local PHEVs can be calculated by the variation of the SOC. Therefore,

$$
\begin{gathered}
x_{i 1}=x_{i 1}^{0}+\left(x_{i 2}-n_{i} x_{i 3}\right)+\left(n_{i} x_{i 3}^{0}-n_{i} x_{i 3}\right) \\
=x_{i 2}-2 n_{i} x_{i 3}+\beta_{i}, \\
\beta_{i}=x_{i 1}^{0}+n_{i} x_{i 3}^{0},
\end{gathered}
$$

where $\beta_{i}$ is a constant.

The equations (5)-(7) are correlated with each other to form an internal energy transformation construction, which constitutes the 3-dimensional state vector of the system. Each region shares the same strength of coupling and activation functions, but with different features. $\beta_{i}$ and $n_{i}$ allow each node to retain its own features.

$f\left(x_{i}(t), n_{i}, \beta_{i}\right)=\left\{\begin{array}{l}\dot{x_{i 1}}=-x_{i 1}+x_{i 2}-2 n_{i} x_{i 3}+\beta_{i}, \\ \dot{x_{i 2}}=-x_{i 2}+n_{i} x_{i 3}, \\ \dot{x_{i 3}}=k x_{i 1} .\end{array}\right.$

The analytical model is used to simulate the process of synchronization and achieve the target SOC. Then, the result of synchronization could direct the experimental model in determining the dynamic price.

\section{Dynamic Price Function}

In the experimental model, PHEVs will adjust their battery level according to the current price.

$$
\text { Battry_level }_{i}=\frac{\beta}{\operatorname{Pr}_{i}(t)},
$$

where $\beta$ is a constant. The target SOC $x_{i 3}$ is the battery level that region $i$ intends to achieve through dynamic price incentives. Therefore, we have the pricing function

$$
\operatorname{Pr}_{i}(t)=\frac{\beta}{x_{i 3}} .
$$

\section{GENERALIZED SynCHRONIZATION}

In this section, we use generalized synchronization with enhanced projective function to achieve system synchronization. 


\section{A. Criterion for Generalized Synchronization}

Definition 1: To achieve generalized synchronization in a V2G network, we propose a new hybrid approach. Based on the model and traditional definition of generalized synchronization, we have

$$
\lim _{t \rightarrow \infty}\left\|H_{i}\left[x_{i}(t), n_{i}\right]-s(t)\right\|=0,(i=1,2, \cdots, N),
$$

where $H_{i}\left[x_{i}(t), n_{i}\right]$ is defined as the enhanced projective function. We consider that there is a unique state $s(t)$ for each node after synchronization. Thus, the solution of the V2G network is $S=\left\{\left(x_{1}^{T}, \cdots, x_{N}^{T}\right)^{T} \in R^{M N}: x_{i}=s(t), \forall i=1, \cdots, N\right\}$. The unique state should satisfy the condition $\dot{s}(t)=\dot{s}_{i}(t)$, for the stability of the synchronization results. In addition, $s_{i}(t)=H_{i}\left(x_{i}(t)\right)$.

We define the solution of the network $S=$ $\left\{\left(x_{1}^{T}, \cdots, x_{N}^{T}\right)^{T} \in R^{M N}: x_{i}=s(t), \forall i=1, \cdots, N\right\}$, where $s(t) \in R^{M}$ is a synchronization solution of each isolated node and satisfying $\dot{s}(t)=\dot{s}_{i}(t)$. In addition, $s_{i}(t)=H_{i}\left(x_{i}(t)\right)$.

As the objective is to balance the energy distribution, we define the synchronized state of an energy system as being energy efficient and balancing energy transportation and consumption. The efficiency of energy utility can be obtained by $x_{i 1}=0$. We define the balance according to (7). In addition, $H: R^{m} \rightarrow R^{M}$ is the continuously differentiable vector map ( $m=3, M=2$ ) and can be presented as

$$
H_{i}\left[x_{i}(t), n_{i}\right]=\left[\begin{array}{ccc}
1 & 0 & 0 \\
0 & -1 & 2 n_{i}
\end{array}\right]\left[\begin{array}{l}
x_{i 1} \\
x_{i 2} \\
x_{i 3}
\end{array}\right]-\left[\begin{array}{c}
0 \\
\beta_{i}
\end{array}\right] .
$$

We use (1) and combine it with the activation function (9). The function of node $i$ can be presented as

$$
\begin{gathered}
x_{11}(t)=-x_{11}(t)+x_{12}(t)-2 n_{1} x_{13}(t)+\beta_{1}, \\
\dot{x_{12}}(t)=n_{1} x_{13}(t)-x_{12}(t)+C \sum_{j=1}^{5} a_{j 1} x_{j}(t) \\
-L \sum_{j=2}^{5} b_{j 1} x_{j}(t), \\
x_{13}(t)=k x_{11}(t) .
\end{gathered}
$$

Then, we use (6) to solve the equation $s_{i}(t)=H_{i}\left(x_{i}(t)\right)$. We have

$$
\left\{\begin{array}{l}
s_{i 1}=x_{i 1} \\
s_{i 2}=-x_{i 2}+2 n x_{i 3}+\beta,
\end{array}\right.
$$

and

$$
\left\{\begin{array}{l}
s_{i 1}=-s_{i 1}-s_{i 2} \\
s_{i 2}=-s_{i 2}+2 n k s_{i 1}
\end{array}\right.
$$

The state vector will be stable only when $s_{i}(t)=s(t)=$ $[0,0]^{T}$. This means that the V2G network system will achieve the stability and the synchronization if all nodes are at the points of zero vector.

\section{B. Stability and Synchronization}

Theorem 1: The V2G network can achieve synchronization when all nodes are in the state $s_{i}(t)$. The solution of $s_{i}(t)$ is a stable result. This means that none of the nodes will deviate from the state $s_{i}(t)$ if they are all in this state. As the consequence, the V2G network will be stable.

Proof: The Jacobian matrix of function (18) is

$$
J=\left[\begin{array}{cc}
-1 & -1 \\
2 n_{i} k & -1
\end{array}\right]
$$

Then, we can get the characteristic equation as

$$
\lambda^{2}+2 \lambda+(1+2 n k)=0 .
$$

Note that $(1+2 n k)>1$, thus $0>\lambda_{1}>\lambda_{2}$. This result demonstrates that the $\mathrm{V} 2 \mathrm{G}$ network system is asymptotic stability and can be stable on the solution of $s(t)=[0,0]^{T}$.

Theorem 2: The system error will convergence to zero, when the V2G network achieve its synchronization.

Proof: The system error of $\mathrm{V} 2 \mathrm{G}$ network is given by

$$
e=H_{i}\left[x_{i}(t), n_{i}\right]-s(t)=H_{i}\left[x_{i}(t), n_{i}\right]
$$

According to (12), we have

$$
\dot{e}=\dot{H}_{i}\left[x_{i}(t), n_{i}\right]=J e .
$$

We know that the Jacobian matrix $J$ is a negative definite matrix. Therefore, the synchronous error $e$ in the $\mathrm{V} 2 \mathrm{G}$ network will approach to zero, and become stable gradually.

\section{NumericAl RESUlTS}

In this section, we verify the proposed analytical model and demonstrate the performance of the proposed price-based control strategy. We use real date in the simulation. The system model is supported by three kinds of data: vehicle travel data, the date of renewable energy generation and the data of local demand. Therefore, we access several database resources to select the typical data that we use in this model.

We adopt the household travel data dictionary of southeast Florida region as the data of the PHEVs' travel [14]. There are three counties in the southeast Florida, Broward, Dade and Palm Beach. Based on the statistics, we find that most of the trips by vehicles taking place inside a single county and only $9.18 \%$ of the travel is crossing county borders. This small part of travel crossing boundary has little impact to the energy dispatch in the system model. Therefore, we only choose Broward as the simulation area. Fig. 3(a) shows the geographic map of Broward. We further divide Broward into 13 parts, and number them from 1 to 13, as Fig. 3(b) has described.

According to the household activity records, most trips happen within two time intervals: in the morning between $6 \mathrm{am}$ and $10 \mathrm{am}$ and in the afternoon between $16 \mathrm{pm}$ and $20 \mathrm{pm}$. Since the energy dispatching is mostly available during rush hours, we conduct our simulations during the first time interval. 


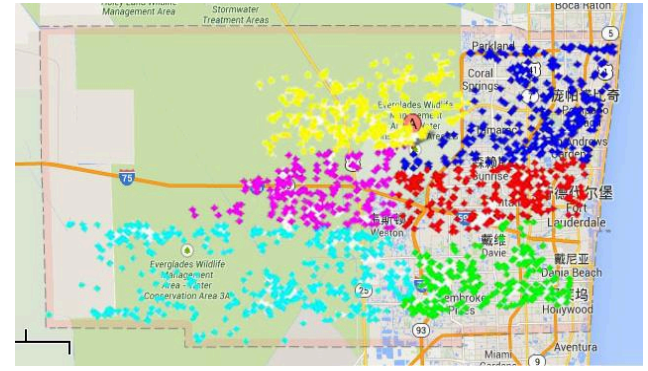

(a) Geographic map of Broward.

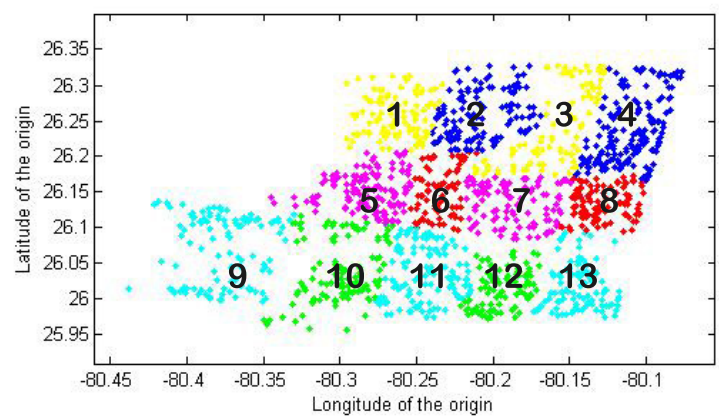

(b) The schematic map of 13 regions.

Fig. 3: The map of targeted area in the experiment.

TABLE I: Load data in the 13 regions in the daytime.

\begin{tabular}{|c|c|c|c|}
\hline Region & $\begin{array}{c}\text { Load demand } \\
\text { (MWh) }\end{array}$ & $\begin{array}{c}\text { The average } \\
\text { load rate }\end{array}$ & $\begin{array}{c}\text { Renewable power } \\
\text { supply(MWh) }\end{array}$ \\
\hline 1 & 37.035 & $80.27 \%$ & 507.69 \\
\hline 2 & 21.837 & $89.70 \%$ & 549.99 \\
\hline 3 & -11.433 & $87.67 \%$ & 0 \\
\hline 4 & 43.131 & $89.96 \%$ & 952.23 \\
\hline 5 & 33.549 & $87.49 \%$ & 676.92 \\
\hline 6 & 2.619 & $75.14 \%$ & 0 \\
\hline 7 & -2.971 & $85.29 \%$ & 0 \\
\hline 8 & 30.237 & $88.53 \%$ & 529.02 \\
\hline 9 & 42.891 & $81.12 \%$ & 592.29 \\
\hline 10 & 47.196 & $79.78 \%$ & 634.62 \\
\hline 11 & -16.161 & $87.49 \%$ & 0 \\
\hline 12 & -0.246 & $80.27 \%$ & 0 \\
\hline 13 & 56.715 & $69.14 \%$ & 740.61 \\
\hline
\end{tabular}

We adopt solar energy data and wind energy data from the daily renewable watch [15]. We refer to the local demand data in the previous study [16]. Table I shows the power load information. It includes the renewable power supply (solar or wind), the average load rate and the load demand in the daytime. The average load rate is calculated by dividing the actual load by the standard load. In this paper, the standard load is $80 \%$ of the maximum load which is regarded as the balance between supply and demand. The load demand is received from the actual load minus the standard load. Positive values mean that the regions need to export amount of energy to other places to achieve balance. Negative values represent the transportation in the opposite direction.

The battery model we used is the Chevy Volts Lithium-ion battery with $15 \mathrm{KWh}$ capability. The nominal charging rate
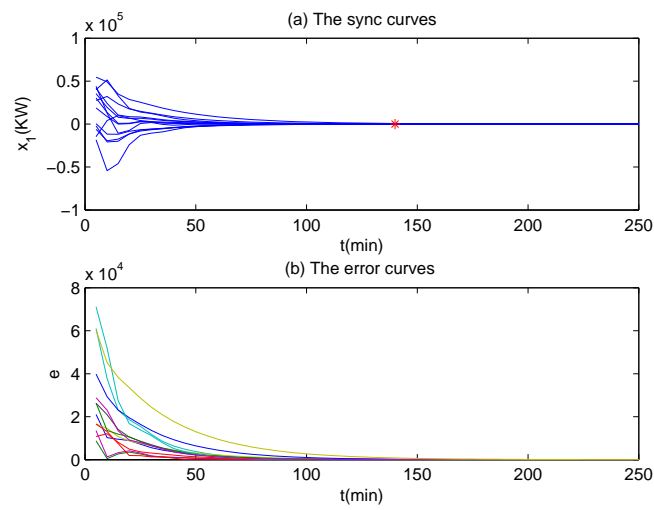

Fig. 4: Synchronization of the V2G network.
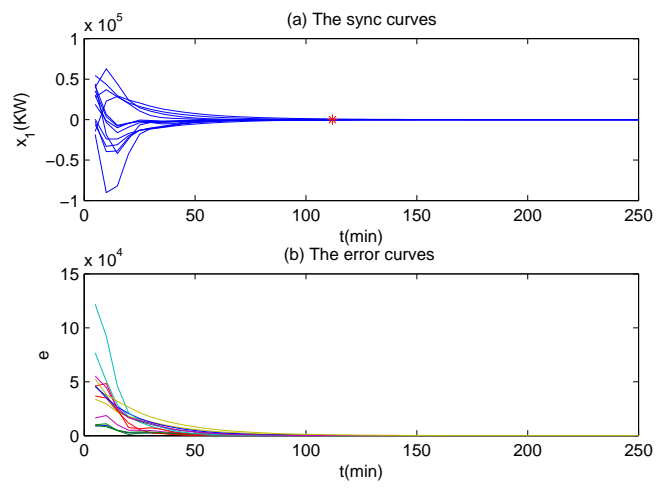

Fig. 5: Synchronization of V2G network at double trip-rate.

is $2.0 \mathrm{KW}$. The parameters of willingness, $\beta=0.8318$. The time horizon is divided by 24 hours a day. We investigate the procedure of synchronization and the results of price-based synchronization control.

We simulate the analytical model in the rush hour because a large number of PHEVs travelling at that time may fulfil the energy transportation needs. We observe the process of synchronization of the V2G network. Fig. 4 shows the synchronization procedure starting at 7 o'clock. Fig. 4(a) is the synchronized curve in the $x_{1}$ component which represents the energy demand level in each region. The error curve is described in Fig. 4(b). We see that the system achieves its synchronization as long as the system error is asymptotically stable to zero.

From Fig. 4, we can observe that the system will achieve its synchronization at time 9:20. Fig. 5 shows the result of doubling the trip rate of the PHEVs at time 7 o'clock and simulates the synchronization process again. Comparison between these two situations indicates that higher PHEVs traffic among different regions speeds up the convergence of the network and reduces the time to reach the synchronization state. When there are more PHEVs, the amount of dispatched energy increases and the time for energy control may be reduced. Such a transportation of power is beneficial with the objective to balance energy supply and demand. Therefore, 


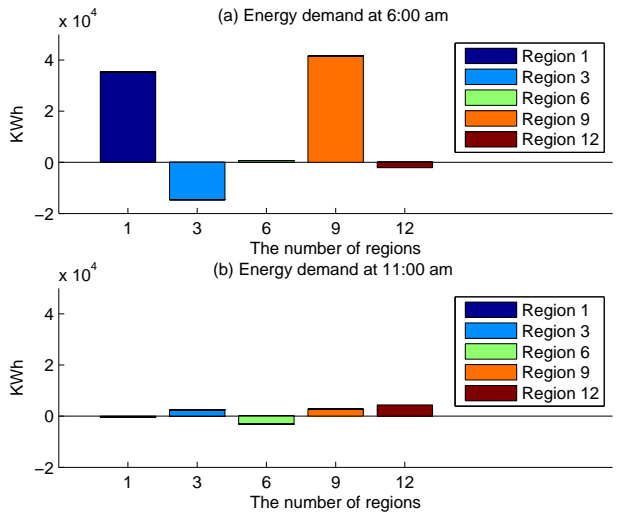

Fig. 6: Energy demand in the morning rush hours.

V2G network synchronization is significant for the efficiency and reliability of industrial smart grid.

Fig. 6 shows the energy demand during the time interval of 6 am to 11 am (the morning rush hours) in regions 1 , 3, 6, 9 and 12. Fig. 6(a) shows the result at time 6:00 am and Fig. 6(b) is at 11:00 am. At the beginning of the rush hours, there is a large difference in energy demand among the regions. At the end of the rush hours, the differences are reduced significantly. We can see that, in some regions the demand for more energy is very close to zero, and in the other regions the demand is very small. The control error is small and caused by some unexpected reasons, such as, PHEVs transportation delay, limited energy transfer rate, and history data difference. could say that the V2G network can perform satisfying energy dispatching under the price-based control with the synchronization method, and hence improve the power grid in balancing energy demand and supply.

\section{CONCLUSION}

V2G network plays an essential role in the industrial smart grid. In this paper, we propose to utilize price-based energy control to dispatch energy among regions. In particular, we take advantage of the PHEV mobility to equivalently transport the renewable energy among regions with different demands. Given a V2G networks with energy state information and history data, the proposed approach could operate adaptively to general network structures or parameter settings. Illustrative results demonstrate that the $\mathrm{V} 2 \mathrm{G}$ networks under the pricebased energy control can achieve energy demand and supply balance in all regions.

\section{ACKNOWLEDGEMENT}

The work is supported in part by programs of NSFC under Grant nos. 61422201, 61370159, 61203117, and U1201253, High Education Excellent Young Teacher Program of Guangdong Province under grant no. YQ2013057, and Science and Technology Program of Guangzhou under grant no. 2014J2200097 (Zhujiang New Star Program).

\section{REFERENCES}

[1] F. R. Pazheri, M. F. Othman, N. H. Malik, T. P. Imthias Ahamed, "Effect of Renewable Penetration on Economic Power Dispatch in Presence of Energy Storage" in Proceedings of 14th IEEE International Conference on Power and Energy, pp. 520-523, 2012.

[2] M. Wolsink, "The research agenda on social acceptance of distributed generation in smart grids: Renewable as common pool resources," Renewable \& Sustainable Energy Reviews, vol. 16, no. 1, pp. 822-835, 2012.

[3] W. Tushar, B. Chai, C. Yuen, D. B. Smith, K. L. Wood, Z. Yang, H. V. Poor, "Three-Party Energy Management with Distributed Energy Resources in Smart Grid," IEEE Trans. Industrial Electronics, June 2014.

[4] A. Saber, G. Venayagamoorthy, "Efficient Utilization of Renewable Energy Sources by Gridable Vehicles in Cyber-Physical Energy Systems," IEEE Systems Journal, vol. 4, no. 3, pp. 285-294, 2010.

[5] S. Hosseini, A. Badri, M. Parvania, "The Plug-in Electric Vehicles for Power System Applications: The Vehicle to Grid (V2G) Concept," in Proceedings of IEEE International Energy Conference and Exhibition (ENERGYCON), pp. 1101-1106, 2012.

[6] R. Deng, J. Chen, X. Cao, Y. Zhang, S. Maharjan, S. Gjessing, "SensingPerformance Tradeoff in Cognitive Radio Enabled Smart Grid," IEEE Transactions on Smart Grid, vol. 4, no. 1, pp. 302-310, 2013.

[7] T. Wu, Q. Yang, Z. Bao, W. Yan, "Coordinated energy dispatching in microgrid with wind power generation and plug-in electric vehicles," IEEE Trans. Smart Grid vol. 4, no. 3, pp. 1453-1463, 2013.

[8] J. Zhou, J. A. Lu, J. Lü, "Adaptive synchronization of an uncertain complex dynamical network," IEEE Trans. Automation Control, vol. 51, no. 4, pp. 652-656, 2006.

[9] Belykh I. V., Lange E., Hasler M., "Synchronization of bursting neurons: what matters in the network topology," Physical review letters, vol. 94, pp. 188-101, 2005.

[10] S. H. Strogatz, I. Stewart, "Coupled oscillators and biological synchronization," Scientific American, vol. 12, pp. 102-109, 1993.

[11] C. G. Li, G. Chen, "Phase synchronization in small-word networks of chaotic oscillators," Physica A, vol. 314, pp. 73-79, 2004.

[12] Z. Zhu, S. Li, H. Yu, X. Liu, "An Approach of Chaotic Generalized Synchronization with the Space Transformation," in Proceedings of IEEE International Symposium on Industrial Electronics, pp. 1088-1092, 2008.

[13] K. Tabata, Y. Kishi, S. Konishi, S. Nomoto, "A study on the autonomous network synchronization scheme for mesh wireless network," in Proceedings of 14th IEEE PIMRC, vol. 1, pp. 829-833, 2003.

[14] Southeast Florida Region Household Travel Survey 1999 Data Dictionary [on-line]:

https://search.yahoo.com/search_ylt=AnKdGB41MTC7q1OMy.cwu.abv $\mathrm{Zx} 4$ ? $\mathrm{p}=$ Southeast+Florida+Region+Household+Travel+Survey+2000+Data + Dictionary \& toggle $=1 \&$ cop $=m s s \& e i=U T F-8 \& f r=y f p-t-443 \& f p=1$.

[15] California ISO, Hourly Breakdown of Renewable Resources [on-line]: http://www.caiso.com/market/Pages/ReportsBulletins/DailyRenewables Watch.aspx.

[16] A. L. Shenkman, "Energy Loss Computation by Using Statistical Techniques," IEEE Transactions on Power Delivery, vol. 5, no. 1, pp. 254$258,1990$. 